(C) [2009] IEEE. Reprinted, with permission, from [Yong Zhang, Guangyuan Yang, K. R. Shao, Youguang Guo, Jianguo Zhu and J. D. Lavers, Multiscale Combined Radial Basis Function Collocation Method for Eddy Currents Analysis in High-Speed Moving Conductors, Magnetics, IEEE Transactions on Volume: 45, Issue: 10, Oct. 2009]. This material is posted here with permission of the IEEE. Such ermission of the IEEE does not in any way imply IEEE endorsement of any of the University of Technology, Sydney's products or services. Internal or personal use of this material is permitted. However, permission to reprint/republish this material for advertising or promotional purposes or for creating new collective works for resale or redistribution must be obtained from the IEEE by writing to pubspermissions@ieee.org. By choosing to view this document, you agree to all provisions of the copyright laws protecting it 


\title{
Multiscale Combined Radial Basis Function Collocation Method for Eddy Currents Analysis in High-Speed Moving Conductors
}

\author{
Yong Zhang , Guangyuan Yang , K. R. Shao , Youguang Guo , Jianguo Zhu , and J. D. Lavers , Fellow, IEEE \\ ${ }^{1}$ College of Electrical and Electronic Engineering, Huazhong University of Science and Technology, Wuhan 430074, China \\ ${ }^{2}$ Faculty of Engineering, University of Technology, Sydney, NSW 2007, Australia \\ ${ }^{3}$ Department of Electrical and Computer Engineering, University of Toronto, Toronto, ON M5S 3G4, Canada
}

\begin{abstract}
A novel multiscale combined radial basis function (RBF) collocation method, as a truly meshless method, is presented to overcome the shortage of general RBF collocation method and is applied to analyze eddy currents in high-speed moving conductors in this paper. A typical example is set here to illustrate the accuracy and affectivity of the proposed method, including a comparison with general RBF collocation method and finite element method (FEM).
\end{abstract}

Index Terms-Eddy current, initial-boundary problem, meshless method, moving conductor, multiscale, RBF.

\section{INTRODUCTION}

$\mathbf{R}$ ADIAL BASIS FUNCTION (RBF) methods, as a truly meshless method for approximating the solutions of partical differential equations (PDEs), have drawn much of the attention of many researchers in science and engineering. In this class of truly RBF meshless methods, the RBF collocation method is ranked the best based on its high accuracy, ease of implementation, good visual aspect, low execution time, and storage requirements. Therefore, it has been used in solving and analyzing three-dimensional (3-D) electromagnetic problems [1] and transient eddy current problems [2].

An obvious characteristic of RBF is that each RBF has its shape parameter $c$, and via it, its shape is only determined. In the RBF collocation method, we adjust the shape parameter to solve PEDs and get accurate solution. Generally, the solutions of PDEs are somewhat flat curves; the RBF collocation method with a suitable shape parameter can get accurate solution. However, in analyzing convection-diffusion problems with high Peclet number, such as solving moving conductor eddy current problems in electromagnetics, RBF collocation method, by choosing a suitable shape parameter, cannot get satisfied solutions because the solutions to these problems are including flat curve part and high-gradient curve part, and no single a shape parameter of a chosen RBF can describe all the characteristics of the solutions. Traditional finite element method (FEM) cannot overcome the oscillations either because that ordinary Galerkin method uses linear-shape functions to approximate the local solution in an element, but in fact the solution is an exponential-type function. Chen proposed a finite analytic element method (FAEM) on analyzing this problem [3]. However, the FAEM is too complex for implementation in practical applications because the method needs obtaining exact solutions of the characteristic equation to any a type of convection-diffusion problem before meshing and solving procedures.

In this paper, a novel multiscale combined RBF collocation method, as a truly meshless method, is proposed to overcome the shortage of general RBF collocation method. It is also applied to analyze eddy current problems in high-speed moving conductors. An example is set in the paper to illustrate the accuracy and effectivity of the proposed method, including a comparison to other numerical methods.

\section{MOVIng CONDUCTOR EDdy CURRENT PROBLEMS}

When electromagnetic field computation involves moving conductors, eddy currents due to the movement of conductors should be taken into account. For the $\boldsymbol{A}-\phi$ method, the governing equations are

$$
\begin{aligned}
& \nabla \times \frac{1}{\mu} \nabla \times \boldsymbol{A}-\sigma\left(\boldsymbol{v}_{\times} \nabla \times \boldsymbol{A}-j \omega \boldsymbol{A}-\nabla \varphi\right)=\boldsymbol{J}_{s} \\
& \nabla \cdot \sigma(\boldsymbol{v} \times \nabla \times \boldsymbol{A}-j \omega \boldsymbol{A}-\nabla \varphi)=0
\end{aligned}
$$

where $\boldsymbol{A}$ and $\varphi$ are magnetic vector potential and electrical scalar potential, respectively; $\mu$ and $\sigma$ are permeability and conductivity, respectively; and $\boldsymbol{v}$ is the velocity of the media relative to the source.

Equation (1.b) is the constrained eddy current equation for (1.a).

For linear conductive medium, with Lorentz gauge that $\nabla$. $\boldsymbol{A}=-\mu \sigma \varphi$, the equivalent form of (1) can be written as follows:

$$
\begin{aligned}
& \nabla^{2} \boldsymbol{A}+\mu \sigma(\boldsymbol{v} \times \nabla \times \boldsymbol{A}-j \omega \boldsymbol{A})=-\mu \boldsymbol{J}_{s} \\
& \nabla^{2} \varphi-j \omega \mu \sigma \varphi=0 .
\end{aligned}
$$

To a moving conductor with uniform speed $\boldsymbol{v}=v \boldsymbol{i}$, define two parameters as

$$
\begin{aligned}
k^{2} & =-j \omega \mu \sigma \varphi \\
2 P & =\mu \sigma v
\end{aligned}
$$

then (2.a) becomes

$$
\nabla^{2} \boldsymbol{A}+2 P \boldsymbol{i} \times \nabla \times \boldsymbol{A}+k^{2} \boldsymbol{A}=-\mu \boldsymbol{J}_{s} .
$$

The typical governing equations of (4) in one-dimensional (1-D) and two-dimensional (2-D) cases are respectively

$$
\begin{aligned}
& \nabla^{2} A-2 P \frac{\mathrm{d} A}{\mathrm{~d} x}+k^{2} A=0 \\
& \nabla^{2} A-2 P \frac{\partial A}{\partial x}-2 P \frac{\partial A}{\partial y}+k^{2} A=0
\end{aligned}
$$




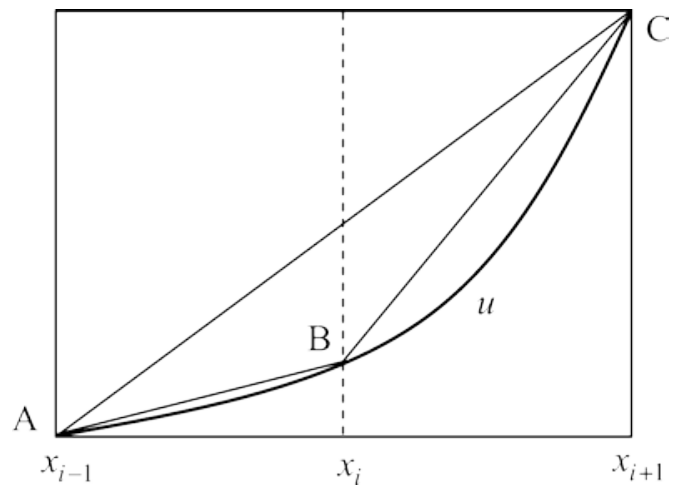

Fig. 1. A portion of exponential function in $i-1 \quad i+1$.

Equations (5.a) and (5.b) are typical convection-diffusion equations. When it is solved by using the ordinary Galerkin finite element method, particularly in the case that the speed $v$ of moving conductors and the relative permeability $\mu$ of the conductors are relatively high, the element Peclet number is typically greater than unity number, and the numerical solutions will contain spurious oscillations [4]. The element Peclet number is defined as

$$
P_{e}=v \sigma \mu h / 2
$$

where $h$ is the length of the element in the direction of the velocity. Therefore, in order to eliminate the spurious oscillations, the mesh must be refined to insure that $P_{e}<1 \quad$ in tradi- tional FEM. This requirement greatly increases the requirement of both the computer memory and the CPU time. In order to solve this problem of oscillations, the upwind methods had great development in the past decades. The upwind scheme uses an unsymmetric weight function, with its upwind side weakened and the downwind side strengthened. This method successfully precludes the spurious oscillations, but introduces excessive dif- fusion. The source of the spurious oscillations is that ordinary Galerkin method uses linear-shape functions to approximate the local solution in an element, but in fact the solution is an expo- nential-type function. The solutions

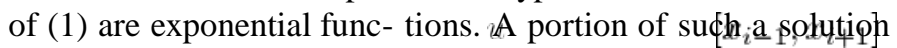
in the interval is shown in Fig. 1.

It is obvious that a linear function only provides a good local approximation to $u$ when the interval is sufficiently small. Fig. 1 also indicates that the derivative of $u$ at the point $x_{i}$ is close to the slope of line $\mathrm{AB}$, the result of a backward difference, rather than the slope of line AC, the result of a central difference. The usual statement-central difference takes with a second-order accuracy, but backward difference only gets a first order accuracy - is not always true without set a presupposition that the interval $\left[x_{i-1}, x_{i+1}\right]$ is sufficiently small. This is the fundamental reason that spurious oscillations appear in ordinary Galerkin solutions of the convection-diffusion equation when $P_{e}>1$. This also helps to understand why upwind method can be considered an artificial modification of linear FEM.

\section{GENERAL RBF COLLOCATION METHOD}

Consider a set of nodes $x_{1}, x_{2}, \ldots, x_{N} \in \boldsymbol{R}^{n}$. The RBFs centered at $\mathbf{x}_{j}$ are defined as

$$
\varphi_{j}(\boldsymbol{x}) \equiv \varphi\left(\left\|x-x_{j}\right\|, c\right) \in \boldsymbol{R}^{n}, \quad j=1,2, \ldots, N
$$

where $\left\|\boldsymbol{x}-\boldsymbol{x}_{j}\right\|$ is the Euclidian norm, and $c$ is shape parameter.

Let $\Omega \subset \boldsymbol{R}^{n}$; we consider a linear partial differential equation with boundary value problem as the form

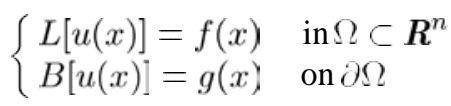

where $\Omega$ is a bounded domain with the boundary $\partial \Omega, L[\cdot]$ is a linear partial differential operator as

$$
\begin{aligned}
& L[\cdot]=\left(\nabla^{2}-2 P \frac{\mathrm{d}}{\mathrm{d} x}+k^{2}\right)[\cdot] \\
& L[\cdot]=\left(\nabla^{2}-2 P \frac{\partial}{\partial x}-2 P \frac{\partial}{\partial y}+k^{2}\right)[\cdot]
\end{aligned}
$$

and $B[\cdot]$ is a boundary operator.

We use $N$ distinct nodes in and on the boundary of $\Omega$, of which $\left\{\boldsymbol{x}_{j}, j=1, \ldots N\right.$ ahd $\left\{\boldsymbol{x}_{j}, j \quad \mathrm{~N}_{I}+1\right.$, are $\boldsymbol{N}$ herior nodes and boundary points, respectively. We look for the approximate solution $u_{h}(x)$ to (8) in the form

$$
u_{h}(x)=\sum_{j=1}^{N} a_{j} \varphi\left(\left\|x-x_{j}\right\|, c\right)=\sum_{j=1}^{N} a_{j} \varphi_{j}(x)
$$

where $\boldsymbol{a}=\left[a_{1}, \ldots, a_{N}\right]$ are unknown coefficients to be determined. Substituting $u_{h}(x)$ into (8) and using collocation at the $N$ nodes, we can get the finite dimensional problem

$$
\begin{aligned}
& \sum_{j=1}^{N} a_{j} L\left[\varphi_{j}\left(x_{i}\right)\right]=f\left(x_{i}\right), \quad i=1, \ldots N_{I} \\
& \sum_{j=1}^{N} a_{j} B\left[\varphi_{j}\left(x_{i}\right)\right]=g\left(x_{i}\right), \quad i=N_{I}+1, \ldots N .
\end{aligned}
$$

This corresponds to the system of equations with a coefficient matrix; its matrix form and solution are

$$
\begin{aligned}
& {\left[\begin{array}{l}
\boldsymbol{L}[\varphi] \\
\boldsymbol{B}[\varphi]
\end{array}\right][\boldsymbol{a}]=\left[\begin{array}{l}
\boldsymbol{f} \\
\boldsymbol{g}
\end{array}\right]} \\
& {[\boldsymbol{a}]=\left[\begin{array}{l}
\boldsymbol{L}[\varphi] \\
\boldsymbol{B}[\varphi]
\end{array}\right]^{-1}\left[\begin{array}{l}
\boldsymbol{f} \\
\boldsymbol{g}
\end{array}\right] .}
\end{aligned}
$$

In (12), we have

$$
\begin{aligned}
\boldsymbol{L}[\varphi] & =\left[\begin{array}{ccc}
L\left[\varphi_{1}\left(\boldsymbol{x}_{1}\right)\right] & \cdots & L\left[\varphi_{N}\left(\boldsymbol{x}_{1}\right)\right] \\
\dot{x_{N}} & \cdot & \cdot \\
L\left[\varphi_{1}\left(\boldsymbol{x}_{N I}\right)\right. & \cdots & L\left[\varphi_{N}\left(\boldsymbol{x}_{N I}\right)\right]
\end{array}\right] \\
\boldsymbol{B}(\varphi) & =\left[\begin{array}{ccc}
B\left[\varphi_{1}\left(\boldsymbol{x}_{N I+1}\right)\right] & \cdots & B\left[\varphi_{N}\left(\boldsymbol{x}_{N I+1}\right)\right] \\
\cdot & \cdots & \cdot \\
B\left[\varphi_{1}\left(\boldsymbol{x}_{N}\right)\right] & \cdots & B\left[\varphi_{N}\left(\boldsymbol{x}_{N}\right)\right]
\end{array}\right] .
\end{aligned}
$$




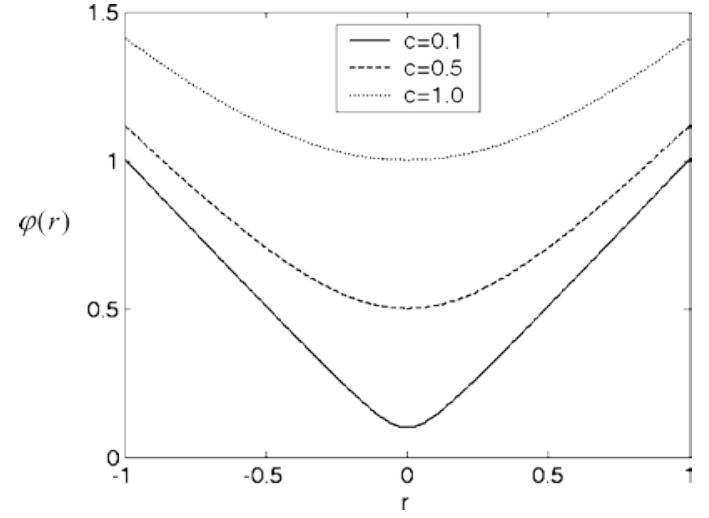

Fig. 2. Diagram of multiquadrics (MQ) under different shape parameters.

RBF is only dependent on spatial coordinates, namely distributed nodes; it has no relationship with any mesh procedure, and this characteristic can make its procedures for solving PDEs simple.

There are three main types of global RBFs usually used in computational electromagnetics as follows:

- Multiquadrics (MQ)

$$
\varphi_{j}(\boldsymbol{x})=\left(\left\|x-\boldsymbol{x}_{j}\right\|^{2}+c^{2}\right)^{0.5} .
$$

- Inverse multiquadrics (IMQ)

$$
\varphi_{j}(\boldsymbol{x})=\left(\left\|\boldsymbol{x}-\boldsymbol{x}_{j}\right\|^{2}+c^{2}\right)^{-0.5} .
$$

- Gaussians (RBF-Gauss)

$$
\varphi_{j}(\boldsymbol{x})=\exp \left(-c^{2}\left\|\boldsymbol{x}-\boldsymbol{x}_{j}\right\|^{2}\right) .
$$

Fig. 2 is the shape diagram of multiquadrics under different shape parameters.

\section{Multiscale Combined RBF Collocation Method}

A classical convection-diffusion equation is set as

$$
\left\{\begin{array}{l}
u^{\prime \prime}-2 P u^{\prime}+k^{2} u=0 \quad \text { in } \Omega \subset \boldsymbol{R}^{n} \\
\left.u\right|_{x=0}=0,\left.u\right|_{x=1}=1
\end{array}\right.
$$

to verify general RBF collocation methods, where $P=$ $v \sigma \mu / 2, P_{e}=P h=v \sigma \mu h / 2$ is the Peclet number, $h$ is nodes scale, and $\sigma$ and $\mu$ are conductivity and permeability, respectively.

There are a total of 21 nodes distributed in the solving domain: 19 nodes for interior and 2 nodes for boundary, respectively. Peclet number $P_{e}=3$ is investigated here to compare the exact solution and the numerical solutions by using MQ collocation method under different shape parameters as described in Fig. 3. It indicates that general RBF collocation methods cannot get good numerical results to moving conductors eddy currents problems as (15).

In the proposed combined RBF collocation method, approximate solution $u_{h}(x)$ are composed with two different types of RBFs as the form

$$
u_{h}(x)=\sum_{j=1}^{N} a_{j} \varphi_{1}\left(\left\|\boldsymbol{x}-\boldsymbol{x}_{j}\right\|, c_{1}\right)+\sum_{k=1}^{N} b_{k} \varphi_{2}\left(\left\|\boldsymbol{x}-\boldsymbol{x}_{k}\right\|, c_{2}\right)
$$

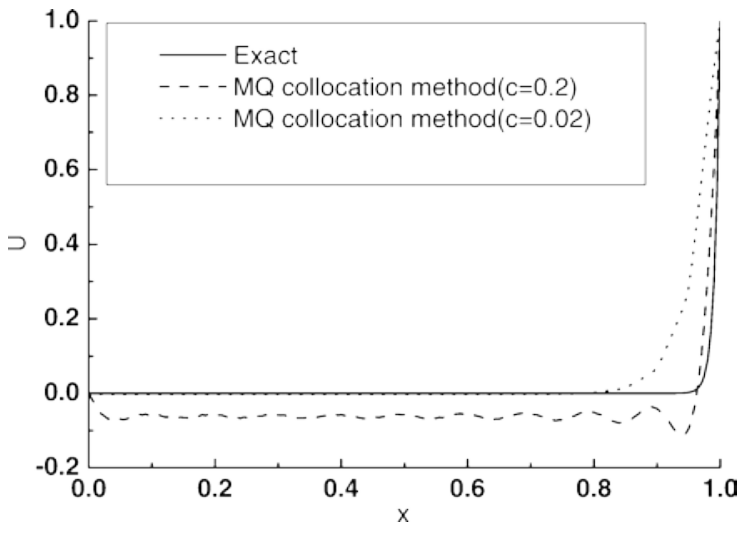

Fig. 3. MQ collocation method solving 1-D moving conductor eddy current problem under different shape parameters.

$$
=\sum_{j=1}^{N} a_{j} \varphi_{1 j}(\boldsymbol{x})+\sum_{k=1}^{N} b_{k} \varphi_{2 k}(\boldsymbol{x})
$$

where $\varphi_{1}\left(\left\|\boldsymbol{x}-\boldsymbol{x}_{j}\right\|, c_{1}\right)$ and $\varphi_{2}\left(\left\|\boldsymbol{x}-\boldsymbol{x}_{k}\right\|, c_{2}\right)$ are different RBFs centered at node $\boldsymbol{x}_{j}$ and node $\boldsymbol{x}_{k}$, respectively, and $\boldsymbol{a}=$ $\left[a_{1}, \ldots, a_{N}\right]$ and $\boldsymbol{b}=\left[b_{1}, \ldots, b_{N}{ }^{\top}\right.$ are unknown coefficients to be determined. Substituting $u_{h}(x)$ into (8), we can get the finite dimensional problem as follows:

$$
\begin{array}{r}
\sum_{j=1}^{N} a_{j} L\left[\varphi_{1 j}\left(\boldsymbol{x}_{i}\right)\right]+\sum_{k=1}^{N} b_{k} L\left[\varphi_{2 k}\left(\boldsymbol{x}_{i}\right)\right]=f\left(\boldsymbol{x}_{i}\right), \\
i=1, \ldots N_{I} \\
\sum_{j=1}^{N} a_{j} B\left[\varphi_{1 j}\left(\boldsymbol{x}_{i}\right)\right]+\sum_{k=1}^{N} b_{k} B\left[\varphi_{2 k}\left(\boldsymbol{x}_{i}\right)\right]= \\
i=N_{I}+1, \ldots N .
\end{array}
$$

This corresponds to the system of equations with a coefficient matrix; its matrix form and system matrix is

$$
\begin{aligned}
& {\left[\begin{array}{ll}
\boldsymbol{L}\left[\varphi_{1}\right] & \boldsymbol{L}\left[\varphi_{2}\right] \\
\boldsymbol{B}\left[\varphi_{1}\right] & \boldsymbol{B}\left[\varphi_{2}\right]
\end{array}\right]\left[\begin{array}{l}
\boldsymbol{a} \\
\boldsymbol{b}
\end{array}\right]=\left[\begin{array}{l}
\boldsymbol{f} \\
\boldsymbol{g}
\end{array}\right]} \\
& \boldsymbol{L}\left(\varphi_{1}\right)=\left[\begin{array}{ccc}
L\left[\varphi_{1}\left(\boldsymbol{x}_{1}\right)\right] & \cdots & L\left[\varphi_{N}\left(\boldsymbol{x}_{1}\right)\right] \\
\dot{x_{N}} & \cdot & \dot{\cdot} \\
L\left[\varphi_{1}\left(\boldsymbol{x}_{N I}\right)\right] & \cdots & L\left[\varphi_{N}\left(\boldsymbol{x}_{N I}\right)\right]
\end{array}\right] \\
& \boldsymbol{L}\left(\varphi_{2}\right)=\left[\begin{array}{ccc}
L\left[\varphi_{2}\left(\boldsymbol{x}_{1}\right)\right] & \cdots & L\left[\varphi_{N}\left(\boldsymbol{x}_{1}\right)\right] \\
\dot{\left.\left.\dot{x}_{N I}\right)\right]} & \cdots & L\left[\varphi_{N}\left(\boldsymbol{x}_{N I}\right)\right]
\end{array}\right] \\
& \boldsymbol{B}\left(\varphi_{1}\right)=\left[\begin{array}{ccc}
B\left[\varphi_{1}\left(\boldsymbol{x}_{N I+1}\right)\right] & \cdots & B\left[\varphi_{N}\left(\boldsymbol{x}_{N I+1}\right)\right] \\
\dot{v} & \cdot & \cdot \\
B\left[\varphi_{1}\left(\boldsymbol{x}_{N}\right)\right] & \cdots & B\left[\varphi_{N}\left(\boldsymbol{x}_{N}\right)\right]
\end{array}\right] \\
& \boldsymbol{B}\left(\varphi_{2}\right)=\left[\begin{array}{ccc}
B\left[\varphi_{2}\left(\boldsymbol{x}_{N I+1}\right)\right] & \cdots & B\left[\varphi_{N}\left(\boldsymbol{x}_{N I+1}\right)\right] \\
\dot{v} & \cdot & \cdot \\
B\left[\varphi_{2}\left(\boldsymbol{x}_{N}\right)\right] & \cdots & B\left[\varphi_{N}\left(\boldsymbol{x}_{N}\right)\right]
\end{array}\right] \text {. }
\end{aligned}
$$

There are $2 N$ unknown coefficients $\boldsymbol{a}=\left[a_{1}, \ldots, a_{N}\right]$ and $\boldsymbol{b}=\left[b_{1}, \ldots, b_{N}\right]$ in (18), but only $N$ nodes for collocation. In 


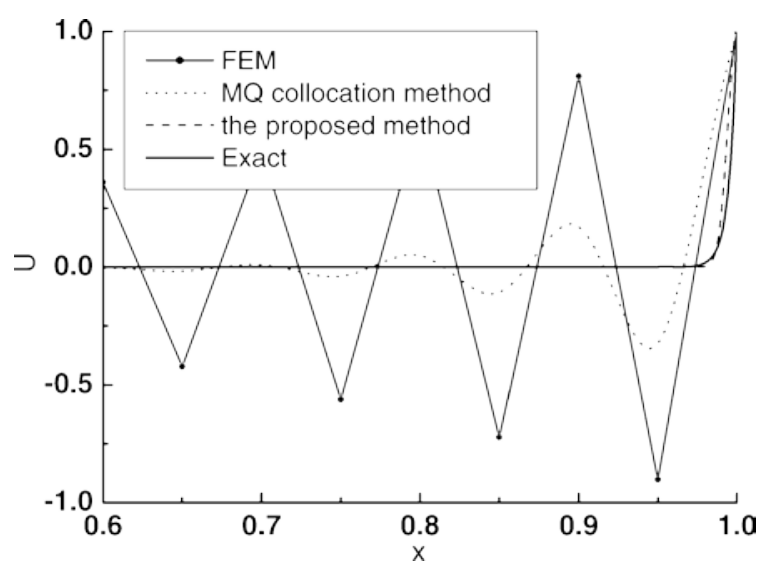

Fig. 4. Comparison of FEM, MQ collocation method, the proposed method, and the exact solutions under

order to determine all the unknown coefficients, we set a constrict condition as

$$
\sum_{j=1}^{N} a_{j} \varphi_{2 j}\left(\boldsymbol{x}_{i}\right)=0, \quad i=1, \ldots N
$$

and its matrix form is

$$
\left[\varphi_{2}\right][\boldsymbol{a}]=[0] .
$$

Combining (18) and (21), a new system matrix can be obtained as

$$
\left[\begin{array}{cc}
\boldsymbol{L}\left[\varphi_{1}\right] & \boldsymbol{L}\left[\varphi_{2}\right] \\
\boldsymbol{B}\left[\varphi_{1}\right] & \boldsymbol{B}\left[\varphi_{2}\right] \\
{\left[\varphi_{2}\right]} & 0
\end{array}\right]\left[\begin{array}{l}
\boldsymbol{a} \\
\boldsymbol{b}
\end{array}\right]=\left[\begin{array}{l}
\boldsymbol{f} \\
\boldsymbol{g} \\
0
\end{array}\right] .
$$

The solution matrix for unknown parameters is

$$
\left[\begin{array}{l}
\boldsymbol{a} \\
\boldsymbol{b}
\end{array}\right]=\left[\begin{array}{cc}
\boldsymbol{L}\left[\varphi_{1}\right] & \boldsymbol{L}\left[\varphi_{2}\right] \\
\boldsymbol{B}\left[\varphi_{1}\right] & \boldsymbol{B}\left[\varphi_{2}\right] \\
{\left[\varphi_{2}\right]} & 0
\end{array}\right]^{-1}\left[\begin{array}{l}
\boldsymbol{f} \\
\boldsymbol{g} \\
0
\end{array}\right] .
$$

\section{NUMERICAL EXAMPLE}

In moving conductors eddy currents analysis for 1-D and 2-D problems, their typical governing equations are described as in (5). No matter in 1-D, 2-D, or 3-D problems, the main difference between moving conductors eddy currents analysis and linear elliptic problems is that the motion of conductors with speed $v$ existed in the former problems, but not in the later ones. Therefore, the classical convection-diffusion equation described in (15) reflects the common nature of 1-D, 2-D, and 3-D moving conductors eddy currents problems. The exact solution for (15) is

$$
u=\frac{\exp [(P+Q) x]-\exp [(P-Q) x]}{\exp [(P+Q) x]+\exp [(P-Q) x]}
$$

where $Q=\sqrt{P^{2}-k^{2}}, \quad$ and and $k$ are defined in (3).

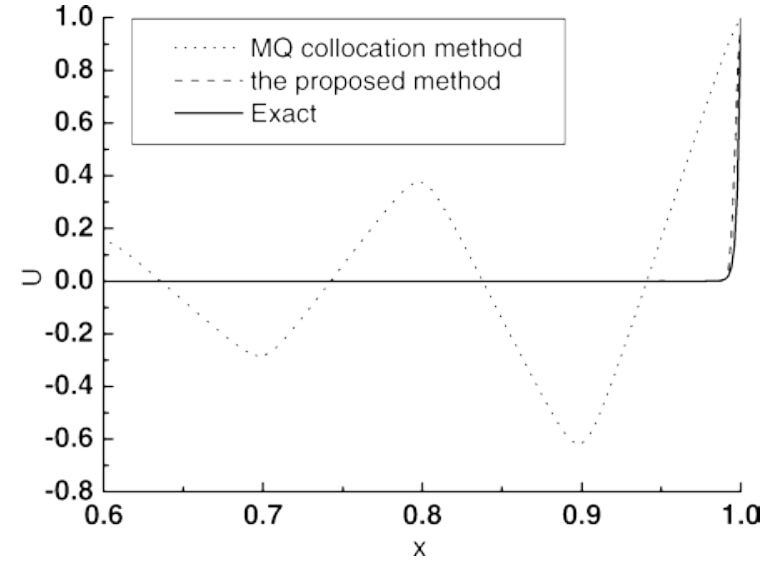

Fig. 5. Comparison of MQ collocation method, the proposed method, and the exact solutions under

We still distribute a total of 21 nodes in the solving domain: 19 nodes for interior and 2 nodes for boundary, respectively. In order to emphasize the impact brought by moving speed $v$ of the conductors, $k^{2}=0$ is set here. The Peclet number $P_{\varepsilon=5}$ and $P_{e}=50 \quad$ are investigated here to compare the exact solu-

tion and the numerical solutions by using the proposed method and other numerical methods. In this paper, MQ from (14.a) and RBF-Gauss from (14.c) are composed as a couple of hybrid RBFs for solving typical governing (15), and $c_{1}$ and $c_{2}$ are shape parameters for MQ and RBF-Gauss, respectively. In the comparison diagram in Fig. 4, shape parameters $c_{1}=0.01$ and $c_{2}=1500$, and in Fig. 5, $c_{1}=0.01$ and $c_{2}=8000$. FEM solution, with spurious oscillations, is omitted in Fig. 5. It indicates that the proposed method is greatly superior to FEM and the general RBF collocation method.

\section{CONCLUSION}

A novel multiscale combined RBF collocation method is presented to analyze eddy currents in high-speed moving conductors. Numerical example proved it is greatly superior to FEM and the general RBF collocation methods. This method, as a truly meshless method, has very large potential for analyzing much more complex 2-D and 3-D moving conductors eddy currents problems.

\section{ACKNOWLEDGMENT}

This work was supported in part by the China Postdoctoral Science Foundation under Grant 20070420170.

\section{REFERENCES}

[1] Y. Zhang et al., "An improved multiquadric collocation method for 3-dimensional electromagnetic problems,” IEEE Trans. Magn., vol. 43, no. 4, pp. 1509-1512, Apr. 2007.

[2] Y. Zhang, K. R. Shao, Y. Guo, J. Zhu, and J. D. Lavers, "Multiquadrics collocation method for transient eddy current problems," IEEE Trans. Magn., vol. 42, no. 10, pp. 3183-3185, Oct. 2006.

[3] D. Chen, K. R. Shao, H. Yu, and J. D. Lavers, "A novel finite analytic element method for solving eddy current problems with moving conductors," IEEE Trans. Magn., vol. 37, no. 5, pt. 1, pp. 3150-3154, Sep. 2001.

[4] N. Burais, N. Foggia, and A. Nicolas, "Numerical solution of eddy currents problems including moving conducting parts," IEEE Trans. Magn., vol. MAG-20, no. 5, pp. 1995-1997, Sep. 1984. 